\title{
SELECTED PROBLEMS IN EXPERIMENTAL INTERMEDIATE ENERGY \\ PHYSICS
}

PROGRESS REPORT

SEPTEMBER 1992

GRANT PERIOD: FEBRUARY 1, 1992 - JANUARY 31, 1993
UNIVERSITY OF HOUSTON

Bill $w$. Mayes

Principal Investigator

Department of Physics

(713) 743-3548

Ed $V$. Hungerford

Principal Investigator

Department of Physics

(713) 743-3549

Lawrence S. Pinsky

Principal Investigator

Department of Physics

(713) 743-3552

Prepared for the

United States Department of Energy

Under contract no. DE-FG05-88ER40419

USDOE Report No. ER-40419-01

DISCLAIMER

This report was prepared as an account of work sponsored by an agency of the United States Government. Neither the United States Government nor any agency thereof, nor any of their employees, makes any warranty, express or implied, or assumes any legal liability or responsibility for the accuracy, completeness, or usefulness of any information, apparatus, product, or process disclosed, or represents that its use would not infringe privately owned rights. Reference herein to any specific commercial product, process, or service by trade name, trademark, manufacturer, or otherwise does not necessarily constitute or imply its endorsement, recommanufacturer, or otherwise the United States Government or any agency thereof. The views and opinions of authors expressed herein do not necessarily state or reflect those of the United States Government or any agency thereof. 
I. Personnel

Personnel who will have worked in support of this contract during the period February 1, 1992 to January 31, 1993 are listed below.
E. V. Hungerford
Professor of Physics
B. W. Mayes
Professor of Physics
L. S. Pinsky
Professor of Physics
L. G. Tang
Research Associate
K. Lan
Research Associate
M. Dzemidzic
Research Assistant
B. Barakat
Research Assistant
J. Flick
Engineer
Y. Lu
Technician
D. Seamon
Technician
z. Wang
Technician
J. Liu
Technician
M. Ahmed
student
D. Lan
student
B. Madaninezhad
student
R. Tully
student
N. Tripathy
student
T. Kelsaw
Secretary 
II. Background

A complete description of the research program of the intermediate energy group at the University of Houston may be found in previous progress reports, renewal proposals, and proposals to the various accelerator advisory committees. Recent documents are appended to this report and summaries of current research activities are listed in the next section. The objectives of this research program are to: 1) investigate forefront problems in experimental intermediate energy physics; 2) educate students in this field of research; and, 3) develop the instrumentation necessary to undertake this experimental program.

Generally, the research is designed to search for physical processes which cannot be explained by conventional models of elementary interactions. This includes the use of nuclear targets where the nucleus provides a many body environment of strongly interacting particles, and where one attempts to observe the perturbation of a known interaction by this environment. Unfortunately, such effects may be masked by the complexity of the many body problem and may be difficult to observe. Therefore, experiments must be carefully chosen and analyzed for deviations from the more conventional models.

There were three major thrusts of the program; 1) strange particle physics, where a strange quark is embedded in the nuclear medium; 2) muon electro-weak decay, which involves a search for a violation of the standard model of the electro-weak interaction; and 3 ) measurement of the spin dependent structure function of the neutron. During this grant period, approximately $60 \%$ of our 
effort has been expended on the MEGA experiment at Los Alamos, a search for lepton number violating decay, $\mu \rightarrow$ er. Approximately $20 \%$ of our effort has been devoted to strange particle physics at Brookhaven and CEBAF, and the remaining $20 \%$ on the SMC experiment at CERN.

The status of our research activities is summarized in Tables I and II. Table I shows those experiments under active investigation, and rable II lists approved experiments and experiments to be undertaken by the research group.

The group has been active in the development of experimental programs at CEBAF. We are interested in the possibility of investigating hypernuclear spectroscopy with an order of magnitude better resolution than currently possible at hadronic machines. This involves the development of a beam line and spectrometer system which would provide the necessary resolution. Finally, we remain interested in the possibility of the development of a high intensity separated kaon and pion beam line in the momentum range of 1-2 GeV/C. This work includes programs at BNL, LAMPF, and KAON where such a line could be constructed. II. Selected Abstracts of Current Research

A. Hyperon Physics at the AGS

The level structure of hyperons embedded in the nucleus (hypernuclei) has been under investigation by our group for some time ${ }^{1}$. The motivation has been to sort out the hyperon-nucleon interaction in the many body system. However, the underlying quark structure in this interaction is just beginning to be addressed. 
Table I. Experiments under Analysis

\begin{tabular}{lll} 
Experiment & \multicolumn{1}{c}{ Description } & Status \\
\hline AGS Exp 773 & Search for an $S=-1$ Dibaryon & Paper published \\
AGS Exp 774 & Search for Sigma Hypernuclei & Data under \\
AGS Exp 828 & $\begin{array}{l}\text { Search for a } 3 \text { Neutron Bound } \\
\text { State }\end{array}$ & analysis \\
AGS Exp 874 & $\begin{array}{l}\text { K Elastic and Quasifree } \\
\text { Scattering }\end{array}$ & Data under \\
LAMPF Exp 969 & Mega & Data under analysis \\
& & Measurement of the Michel \\
LAMPF Exp1240 & Parameter Rho & Data under
\end{tabular}


Table II. Approved Experiments

\begin{tabular}{|c|c|c|}
\hline Experiment & Description & Status \\
\hline LAMPF Exp 969 & MEGA & $\begin{array}{l}\text { Approved } \\
\text { (running) }\end{array}$ \\
\hline CERN EXP NA47 & $\begin{array}{l}\text { Spin Dependent structure } \\
\text { Functions of the Neutron and } \\
\text { Proton }\end{array}$ & $\begin{array}{l}\text { Approved } \\
\text { (running) }\end{array}$ \\
\hline $\begin{array}{r}\text { CEBAF Exp } 9 \\
\end{array}$ & $\begin{array}{l}\text { Spin Dependence of } p \text { shell } \\
\text { Hypernuclei }\end{array}$ & $\begin{array}{l}\text { Approved } \\
\text { (run 1994-95) }\end{array}$ \\
\hline BNL EXP 874 & $\begin{array}{l}\text { Kaon Nucleus Quasielastic and } \\
\text { Elastic Scattering }\end{array}$ & $\begin{array}{l}\text { Approved } \\
\text { (running) }\end{array}$ \\
\hline LAMPF Exp 1240 & $\begin{array}{l}\text { Measurement of the Michel } \\
\text { Parameter RHO with the Mega } \\
\text { Position Spectrometer }\end{array}$ & $\begin{array}{l}\text { Approved } \\
\text { (running) }\end{array}$ \\
\hline BNL Exp 887 & $\begin{array}{l}\text { Do Narrow } \Sigma \text { Hypernuclear } \\
\text { States exist }\end{array}$ & $\begin{array}{l}\text { Approved } \\
\text { Run } 1993-94\end{array}$ \\
\hline
\end{tabular}


It was suggested some time ago ${ }^{2}$ that by placing a hyperon into what would otherwise be an indistinguishable sea of up and down quarks, unique configurations of the strange quark due to the interaction of the pauli principle may provide the key to unlock the quark picture of the aucleus. For example, one would like to place a lambda deeply within the nucleus and determine the level structure with high accuracy. Changes in this structure due to partial deconfinement of the quarks in the lambda may occur. Alternatively a change of the size of the lambda in the nucleus may also explain lambda-sigma coupling ${ }^{3}$ or perhaps three body forces ${ }^{4}$ This could also be connected to the significant charge symmetry breaking observed in the lambda-nucleon interaction ${ }^{5}$.

Experiment 820 was a search for the lower energy member of a proposed spin zero strangeness -1 dibaryon doublet ${ }^{6}$. These data comprised the phd dissertation of a University of Houston graduate student, $K$. Jonhnston. The dibaryon would have spin zero, ind $1=1$ angular momentum, lying just below the $\Sigma-N$ threshold. To excite this state via the $(K, \pi)$ reaction, the di-proton in a ${ }^{3}$ He target is used to obtain a spin singlet nucleon pair ${ }^{7}$. With this target the residual neutron is regarded as a spectator to the reaction. Thus the reaction is ${ }^{3} \mathrm{He}(K, \pi) D_{s} n$. The results of the experiment show that if a dibaryon is present it lies under the backgrounds, so a detailed understanding of background shapes, if not their magnitudes, is necessary. We have treated the backgrounds as arising from three independent amplitudes added incoherently. Above threshold we assume that the background is due to the - 
quasifree production of from a proton in the He target. The shape of this background is obtained using the $\Sigma$ elastic peak from the liquid hydrogen target calibration runs, with the same analysis cuts as for the ${ }^{3}$ He target. The $\Sigma$ peak from this spectrum is then spread by the Fermi momentum of a proton in ${ }^{3}$ He as determined from a Fourier transform of a ${ }^{3} \mathrm{He}$ ground state wave function ${ }^{8}$.

Below threshold the dominant background comes from the two step lambda production on the di-protion in the ${ }^{3}$ He target ${ }^{9}$. The spectrum shape is represented by the shape of the one step ${ }^{3} \mathrm{He}\left(K, \pi^{-}\right)$ spectrum again measured under the same experimental conditions. The assumption here is that both spectrum shapes are dominated by the interactions of the final state particles which are the same within isospin. It is of course possible to produce a dibaryon in both reactions, but the dibaryon to background ratio for $\left(K, \pi^{-}\right)$ reaction would be much smaller than for the two step reaction, so little is lost in treating the experimental $\left(K, \pi^{-}\right)$spectrum as background.

The third piece of the background spectrum is due to the cusp and virtural $\Sigma N=\Lambda N$ conversion. This peak, as described earlier, lies near the $[N$ threshold with a tail running toward lower mass. A representation of this is obtained by calculating the $\Sigma_{N}$ production amplitude using the complex scattering length expansion. This approximation is valid near threshold where the amplitude is maximal, and has been shown to give a reasonable representation of the cusp region ${ }^{10}$. The amplitude must be integrated over the unobserved neutron momenta which is treated as a spectator. 
These three processes are added incoherently and fit to the data via a chi square minimization procedure, varying the three normalization strengths. The result of this fit is shown in Figure 1. The fit gives chi square per degree of freedom of 1.1 , so within the statistical accuracy of the data, no additional strength is needed. Thus to a level of about $0.5 \mu \mathrm{b} / \mathrm{s} r$ no dibaryon signal is evident.

The data from Exp. 774 comprised the phd dissertation of one of our graduate students, M. Barakat. Previously several enhancements, identified as $\Sigma$ hypernuclei have been experimentally studied ${ }^{11}$. These data concerning narrow, and in particular bound, states of $\sum$ nuclear systems, were statistically inconclusive. Because a $\Sigma$, whon placed in a nuclear medium, can undergo a strong conversion $\Sigma N=\Lambda N$, the issue turns on whether there is some mechanism by which this process can be suppressed in a nucleus. such a mechanism, called selectivity, has been proposed ${ }^{12}$. It uses the fact that experimentally the $\Sigma N=\Lambda N$ reaction appears to be greatly suppressed in the spin singlet, isospin $3 / 2$ initial state. Therefore, if the $\Sigma$ is formed with low momentum transfer on a nucleus, it can acquire the spin and orbital momentum quantum numbers of the nucleon it replaces, and since the nucleons in the nucleus are highly correlated, one should expect that the conversion width of some states would be suppressed.

The Lest targets for testing the selectivity mechanism are the s shell isotopes. P shell hypernuclei would generally produce too many levels, some broad, which would make it difficult to detect 


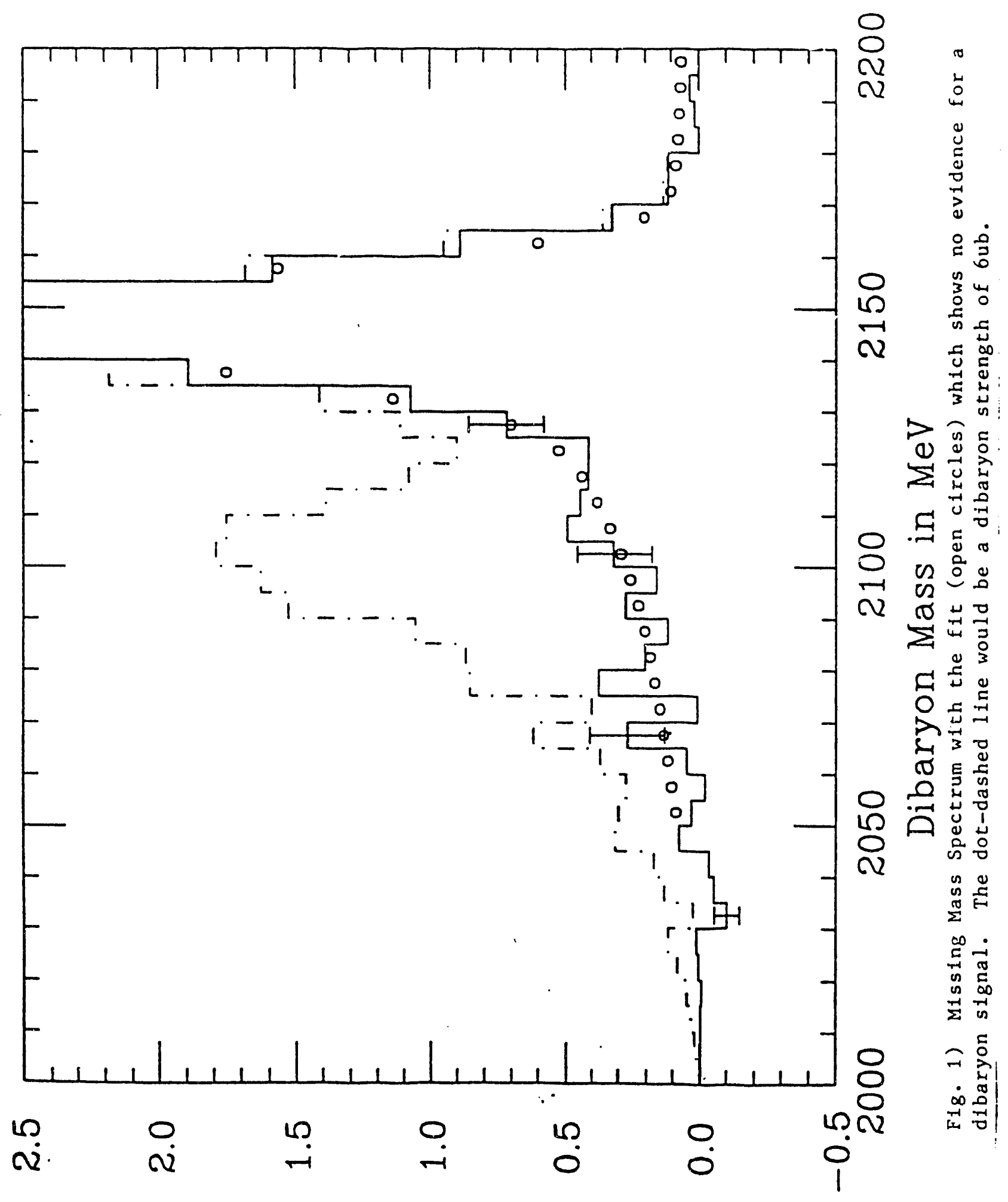

$\Lambda^{2} \mathrm{~K} 0^{\circ} \mathrm{g} / \mathrm{As} / \mathrm{qh}$ uṭ uoțoas ssoxo 
Width faciors $Q_{a}(T, S)$ for the $\Sigma N N$ system

\begin{tabular}{|c|c|c|c|c|}
\hline$T$ & $S$ & $T_{c}$ & $S_{c}$ & $Q_{a}(I, S)$ \\
\hline 0 & $1 / 2$ & 1 & 0 & 3 \\
\hline 1 & $1 / 2$ & 0 & 1 & $1 / 3$ \\
\hline 1 & $3 / 2$ & 0 & 1 & $1 / 3$ \\
\hline 1 & $1 / 2$ & 1 & 0 & 2 \\
\hline 2 & $1 / 2$ & 1 & 0 & 0 \\
\hline
\end{tabular}

Width Factors $Q_{\alpha}(T, S)$ for the $\sum N N N$ System

\begin{tabular}{|c|c|c|}
\hline$T$ & $S$ & $Q_{0}(T, S)$ \\
\hline $1 / 2$ & 0 & 2 \\
\hline $1 / 2$ & 1 & $14 / 9$ \\
\hline $3 / 2$ & 0 & 0 \\
\hline $3 / 2$ & 1 & :8/9 \\
\hline
\end{tabular}

Table III. Width Factors for s Shell Sigma Hypernuclear Systers $Q$ is the width suppression factor 
narrow structure, Table III gives the predicted width factors for the $\Sigma N N$ and $\Sigma N N N$ systems ${ }^{12}$. There is one state in each system where the conversion width is completely suppressed. However, spinisospin mixing in the $\Sigma N$ interaction would dilute the suppression effect. In addition, the residual interaction also splits the energy levels. Thus, the $I=2, S=1 / 2$ state in $\Sigma N N$ is shifted toward less binding and probably forms an unbuund sys'em since the $\Sigma$-nuclear potential is rather shallow. On the other hand, the $I=1$, $S=1 / 2$ state shifts downward and the potential becomes substantially more attractive. The width of this state is suppressed by $1 / 3$ so it may attain a natur..i width of a few Mev.

On the other hand recent results with stopped kaons at KEK have suggested a bound $\Sigma$ state in $\Sigma^{+}$NNN and no state in $\Sigma^{-} N_{N N^{13}}$. A repulsive core was added to the $\Sigma$ nucleus potential to produce a narrow widths for the $I=1 / 2, S=0$ state, which would be broad in the selectivity model. Experiment 774 acquired data for both ${ }^{4} \mathrm{He}$ and ${ }^{3} \mathrm{He}$ targets, investigating both $\left(K, \pi^{+}\right)$and $\left(K, \pi^{-}\right)$reactions. The University of Houston was responsible for the analysis of the $A=3$ data.

The target was surrounded by a scintillation hodoscope which provided a rough measure of the azimuthal and polar angles of decay particles emitted from the target after the reaction. Although the angular information was not particularly helpful, the multiplicity of particles emitted after production helped to determine the configuration of the recoiling hypernulceus. Thus a multiplicity 1 event (one decay particle) is most likely a pion from free $\Sigma^{-}$ 
decay. This would represent $\Sigma$ quasifree production. A multiplicity 2 event is most likely due to a $\wedge$ decay that occurs from $\Sigma N \rightarrow \Lambda N$ conversion, or due to $\Lambda^{\prime} s$ from $\Sigma^{n}$ decay which are produced from $\Sigma^{-}$charge exchange on other nucleons. Fig. 2 is an excitation energy spectrum cut by multiplicity two.

We attribute the strength below $\tau^{-}$-deuterium threshold to the formation of $\Sigma^{\circ}-\mathrm{NN}$. This conclusion is supported by the fact that no such structure is seen in the multiplicity 2 spectrum where both particles penetrate the hodoscope barrel. Any $\Gamma^{\prime} s$ converting on a nucleon producing $\Lambda^{\prime} s$ could generate such an event. Thus we conclude that the ${ }^{3} \mathrm{He}\left(\mathrm{K}^{-}, \pi^{+}\right) \mathrm{X}$ data show no evidence of a $\Sigma^{-}$bound state, and the strength below threshold is consistent with the formation of the $\Sigma^{0} n n$ system. Also the $\Sigma N \rightarrow \Lambda N$ conversion is most probable around the $\Sigma$ production threshold region where one would expect the $\boldsymbol{I}$ to have the lowest possible energy. The conversion probability decreases with the increase of excitation energy since more energy is then available to the $\Sigma$ to escape the recoiling nuclear system. Finally as a consequence of these results the validity of the spin and isospin selectivity mechanism is in question here. Several assumptions were made in constructing the model. The first is that isospin is a good quantum number, and the second is the form and strength of the $[$-Nuclear core potential. Obviously at least one of these assumptions is not appropriate for the $A=3$ system.

Experiment 874 is a study of quasielastic and elastic scattering begun during this past AGS cycle. Recent theoretical 


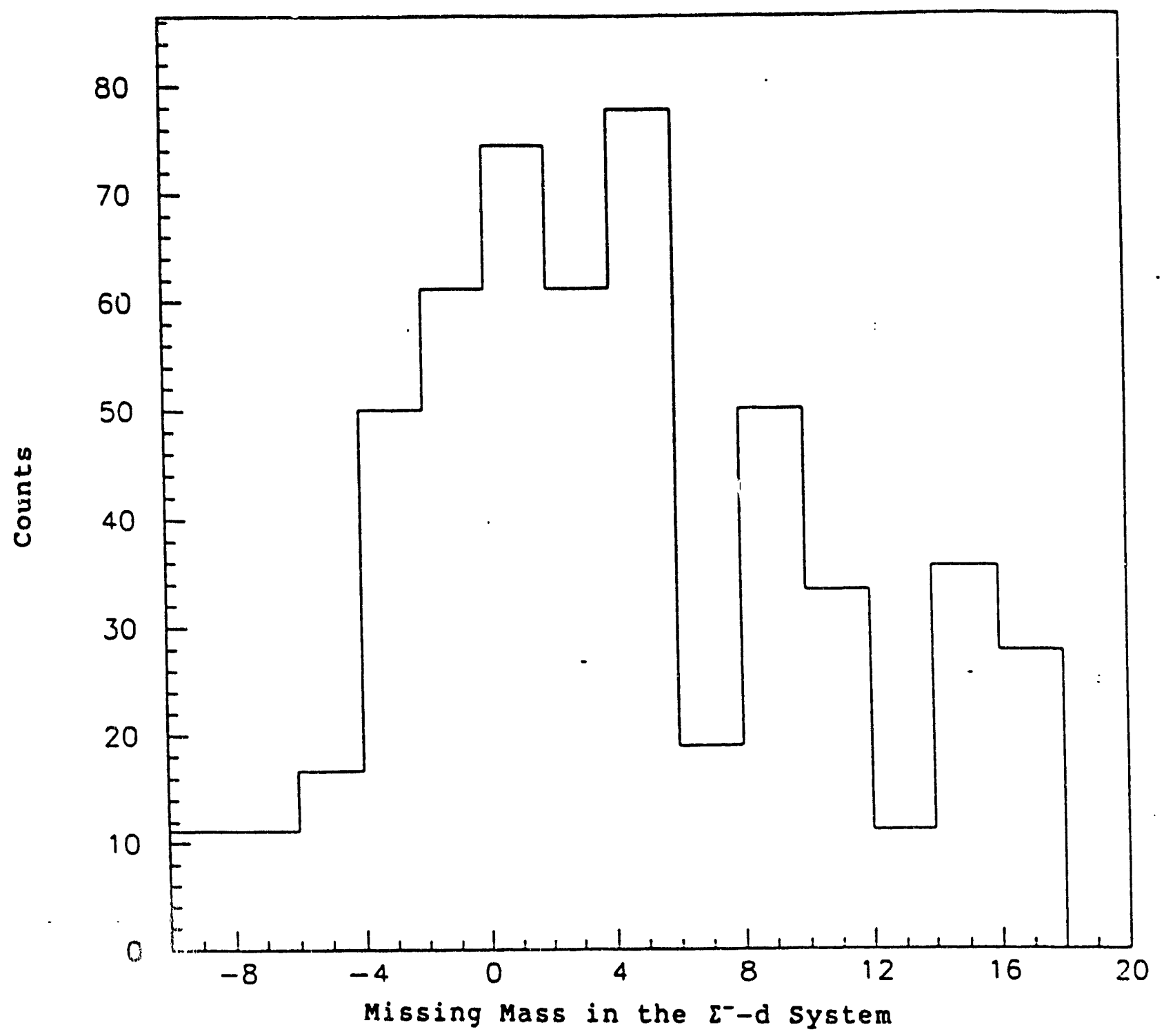

Figure 2) Missing Mass Spectrum cut by multiplicity 2 showing the strength near just below the $\Sigma^{-}-d$ threshold 
work suggests that the $K^{+}$nucleus interaction should be sensitive to changes in the structure of a nucleon when that nucleon is embedded in the nucleus ${ }^{14}$. Motivation for this suggestion comes from the realization that the $\mathrm{K}^{+}$nucleus interaction is weak and thus volume dependent. However $\mathrm{K}^{+}$elastic scattering data on $\mathrm{C}$ and Ca at $800 \mathrm{MeV} / \mathrm{c}$ cannot be fitted with the best microscopic optical models, even though the multiple scattering series has been shown to converge rapidly ${ }^{15}$. More recently, total cross section data shows an enh ncement over conventional calculations, although not quite like that predicted by the theory ${ }^{16}$. On the other hand the elastic datal7 has a normalization error of $\pm 17 \%$, and could be brought inta agreement with more conventional models if the data rather than the theory were rescaled ${ }^{18}$. While a rescaled nucleon size is also suggested by other experiments, in particular the "EMC effect", it is important to base these concepts on a variety of diverse experiments.

If Kaons have a long mean free path, incoherent quasielasctic scattering of positive kaons should be proportional to the number of target nucleons in isossymmetric $(T=0)$ targets. In this case, one can search for evidence of alterations to the free characteristics of nucleons when these nucleons are placed in the interior of a nucleus by precision measurement of quasielastic scattering on several $\mathrm{T}=0$ targets with varying volume to surface ratios.

A different approach to rescaling was presented in ref. 19. Here it was assumed that the meson masses within the nuclear medium 
decrease with increasing nuclear density. They point out that $k$ scattering is similar to electron scattering in that at least two pions or a vectro meson must be exchanged in first order. Since the effective radius of the nucleon in the nuclear environment is not only due to the "bare" nucleon radius but also the range of the vector meson, the effective radius should be density dependent. The theoretical result gives a similar description to those with a rescaled nucleon radius. However the authors point out that the results are density dependent, and also depend on the isospin of the target, because the exchange of a $\varphi$ or an $\omega$ is isospin dependent. A similar study has also been made for proton scattering with similar conclusions ${ }^{20}$. This work also ties the anamolous $k$ scattering to the "EMC effect".

Therefore we have proposed (AGS Exp. 874) to investigate $K$ elastic and quasifree scattering from nuclear systems to provide more accurate data to compare to theory, and to investigate possible momentum dependence in the scaling parameters to better. reconcile the theory with the recent total cross section measurements. The proposal calls for $a \pm 58$ error in the elastic cross section normalization.

The experiment was partially completed this past year. Data were obtained on quasifree proton knockout by $\mathrm{K}^{+}$mesons or nuclear targets ranging from $\mathrm{Li}$ to $\mathrm{Pb}$. The data are to be compared to quasifree scattering from deuterium. Data were acquired at 720 $\mathrm{MeV} / \mathrm{C} \mathrm{K}^{+}$momentum. In addition a angular distribution of $\mathrm{K}^{+}$ scattering from a $C$ target at $720 \mathrm{MeV} / \mathrm{C}$ was obtained. These later 
data are parts of the elastic portion of the experiment which will require another 300 to $400 \mathrm{hrs}$. to complete. Preliminary spectra are shown in Fig. 3 .

B. Electroproduction of Hypernuclei

A Hypernucleus is a many body system comprised of conventional (nonstrange) nucleons and one or more strange baryons or hypernucleus adds a new dimension to our evolving picture of nuclear physics. At the hadron level, such hypernuclei provide a new many body spectroscopy, where dynamical symmetries may appear which are forbidden in ordinary nuclei by the pauli principle. Thus a hypernucleus provides a laboratory in which to explore properties af the $\Lambda$-nucleon interaction in the nuclear medium.

On the elementary interaction level, several novel features of the hyperon-r.an interaction play a significant role in hypernuclear physics ${ }^{21}$. Because of isospin the $\Lambda$ and the Nucleon cannot exchange one pion, so there is no dominant OPE tensor force as exists in the nucleon-nucleon interaction. (The $K$ and $K^{+}$ exchange tend to cancel more completely than do the $\pi$ and $P$ exchange in the NN force). This may contribute to the anomalously small binding observed in $\Lambda+\mathrm{He}^{4}$ system $^{22}$. The absence of a direct OPE force ensures that shorter range properties of the baryonbaryon interaction are important in hypernuclei. The two pion exchange, which is overshadowed by the OPE in the NN force, is the major long-range component of the interaction. Because the $\Lambda-\Sigma$ mass difference is only some $80 \mathrm{MeV}$, the $\Lambda$ and the $\Sigma$ couple more strongly than do the nucleon and $\Delta$ in the nonstrange sector. This 


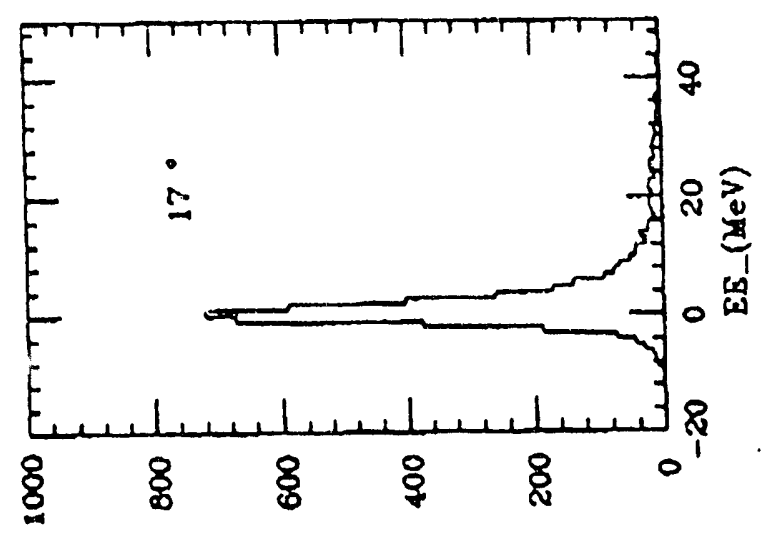

$\Lambda$ กห/8junos

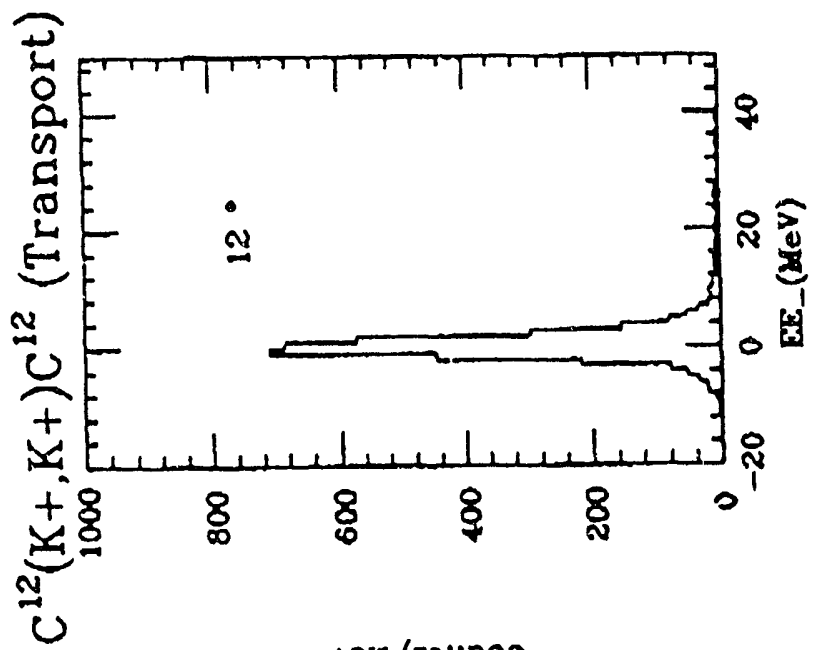

son/squnos

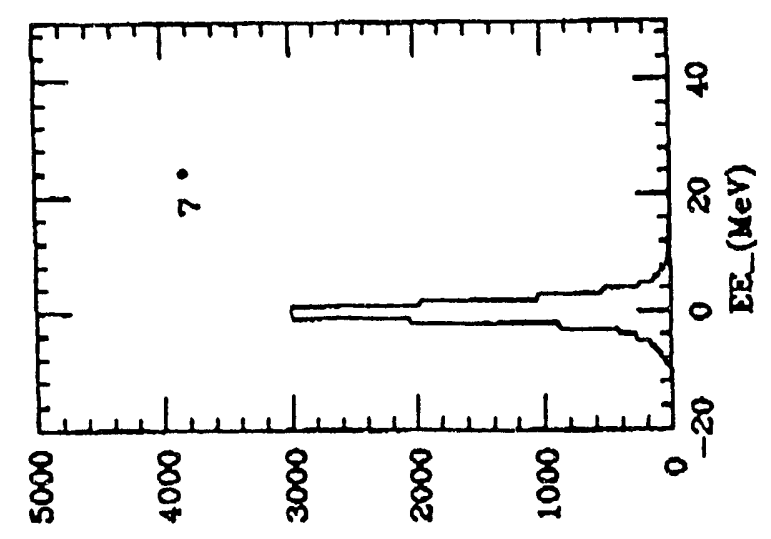

nok/spunos

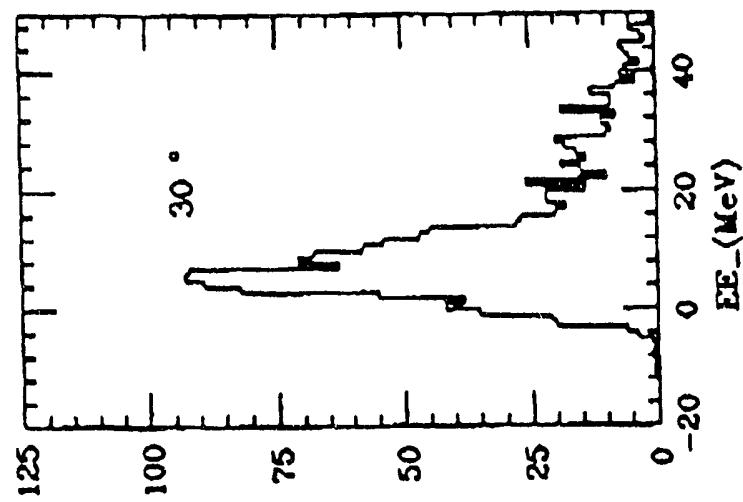

ser/ozunos

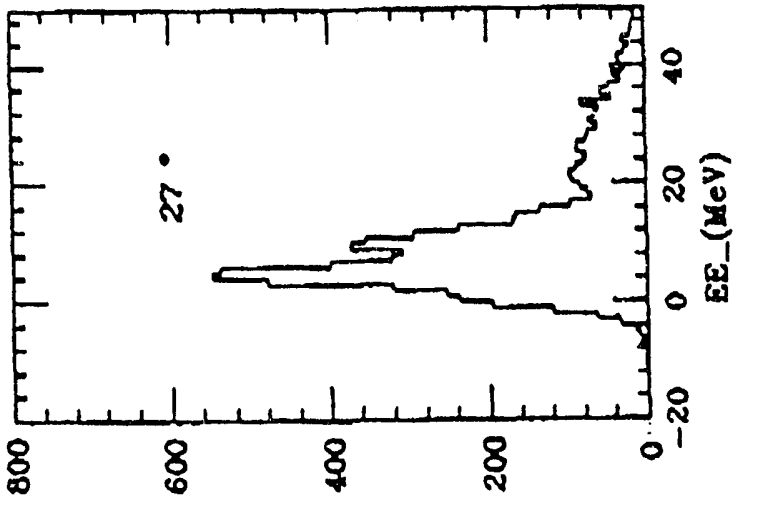

heN/Equnos

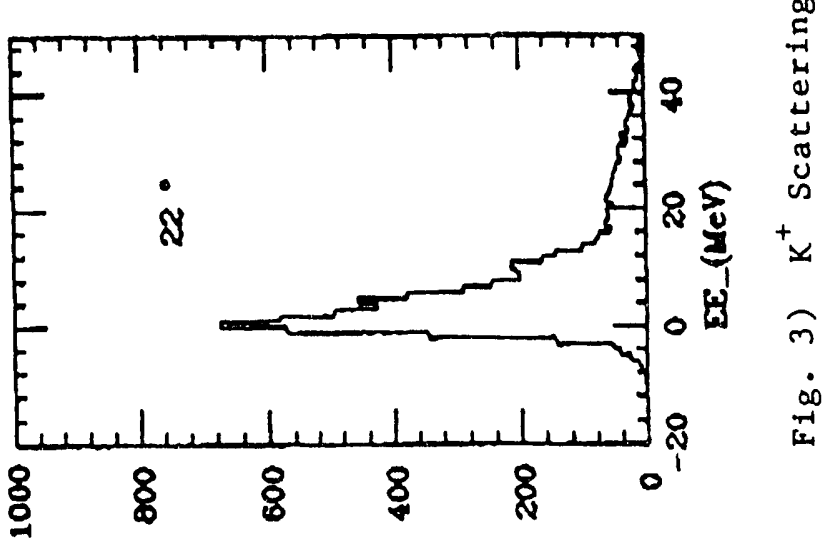

$\Lambda$ ก/egunos 
strong coupling leads to a nonnegligable second order tensor force in the $\wedge$-nucleon channel, and to sizeable three-body forces. The strong coupling, combined with a sizable $\Sigma^{-}$to $\Sigma^{+}$mass difference and the charge dependence in the $\Sigma$-nucleon OPE force, imply a measurable charge symmetry breaking in the hyperon-nucleon interaction. A large charge asymmetry is in fact observed in the $A=4$ mass isodoublet ${ }^{23}$. Thus novel aspects of the hyperon-nucleon interaction produce observable effects in $\wedge$ hypernuclei. The $\wedge$ as a distinguishable baryon, resides in the $1 \mathrm{~s}$ shell for the hypernuclear ground state. Broad structure in the spectra of light systems has been observed. The significant physics lies in the fine structure of those spectra which requires a high-intensity, high-resolution facility to exploit.

Electromagnetic production of hypernuclear levels will strongly populate unnatural parity states starting from a $j=0+$ nuclear target ${ }^{24}$. This contrasts to the hadronic production mechanisms where the spin flip amplitude is small. In addition, sir.e the momentum transfer is large, one expects to selectively excite states with high angular momentum transfer. Therefore CEBAF can not only provide high resolution data, but it also provides complementary data to hadronic production reactions ${ }^{25}$.

We have proposed an experiment at CEBAF (Exp. 9), now approved to investigate the electromagnetic production of hypernuclear states with high resolution $(200 \mathrm{keV})$. This experiment will first use the sos spectrometer in Hall C for kaon detection and will give experimental resolutions on the order of $1 \mathrm{MeV}$. 
However, we are developing a kaon spectrometer design based on the concept that resolution in the resulting hypernuclear system is to be optimized. The kaon and electron spectrometers are to be placed at forward angles $\left(>5^{\circ}\right)$. This also allows thin samples and reduced energy loss which is also important to the resolution. At forward angles, the count rate is limited by the electron spectrometer, and one expects a relatively low hadronic background in the kaon spectrometer. This facilitates detectors employing particle identification, as well as hadionic tagging methods for background and quasi-free suppression of the resulting spectrum.

C. Test of the standard Model of Electro-weak Interactions ( LAMPF .969)

The experiment acronym, MEGA, is derived from the first letter of the branching ratio, $u=e r$, which is to be investigated in this experiment. The experiment is designed to be a background free measurement of this particular branching ratio to one part in $10^{13}$. Presently, it appears that the experiment can achieve a limit of approximately $2 \times 10^{13}$. A measurement to this accuracy would be about a factor of 250 better than the present experimental limit ${ }^{26}$. The experiment tests the standard model of electro-weak interactions, and may be interpreted in terms of a model characterized by new particles. In this measure, MEGA will raise the mass scales of new particle searches by almost an order of magnitude. MEGA is a collaborative effort of 11 institutions. The University of Houston in direct association with Texas A\&M University is responsible for the construction of the photon 
detector.

This detector consists of 3 layers of cylindrical detectors with each layer serving as an independont detector and having the same geometry except for the radial dimensions. The two lead converters are separated by an MWPC to identify in which converter the lepton pair was produced, and the three drift chambers are used to track the conversion pair to determine the energy of the photon. In the smallest layer, there are 416 MWPC wires and 624 DC wires. Position perpendicular to the wire, albeit within the well known left-right and multiple hit ambiguities, can be determined by the drift time of the ionized charge. Position alcng the wire ("z" position) is. not so easily obtained. Normally stereo wires, stereo cathode readout foils, and charge division are methods of choice ${ }^{27}$. In the MEGA photon detector we use a delay line readout technique to determine "z" position" ${ }^{28}$. The chambers are constructed using low mass, high strength composite materials (carbon fibers). Tolerances require that the cylinders form perfectly cylindrical surfaces to within $150 \mu$ on a $1.5 \mathrm{~m}$ diameter. This precision involves not only careful machining techniques, but it also requires constant temperature control during fabrication and operation.

Readout electronics for the detector system uses an application specific IC (ASIC) hybrid preamplifier designed by our group and manufactured by Phillips Circuit Assemblies. The amplifier/discriminator is also a hybrid ASIC. The computer interface electronics is based on FASTBUS using a CHI controller 
and DEC station nodes. Each node is used to filter the data before storage in a MICROVAX III and on magnetic tape. The data acquisicion system is designed to operate in a high rate, high background environment, selecting only those events which satisfy a specific track pattern. The data stream will be multiplexed (actually "or'ed") before entry into FASTBUS to reduce the number of FASTBUS channels.

Two of the three photon layers were installed this past year in the LASS magnet at LAMPF. These detectors were checked with both cosmic rays and beam. They performed well with both field on and field off measurements of efficiency and resolution. We were particularly gratified that the MWPC detector worked so well, since it was the most critical to operate and the detector with which we had problems in the past. This year we used a fast drift gas especially developed and tested by our group for fast charge collection and stability. Fig. 4 shows efficiency of the drift chambers tested by cosmic rays.

In addition to testing and calibration of the detectors, the last two weeks of the run cycle at LAMPF were used to collect $\mu \rightarrow e r$ data triggers. The about $3 \times 10^{12} \mu$ stops were collected with approximately 808 of them on tape. While it is not clear what the overall efficiency of the system will be for $\mu \rightarrow e r$, we expect that this year's run will provide a branching ratio near the present limit of $5 \times 10^{-11}$. In addition to the $\mu \rightarrow e r$ data we also have data on the measurement of the Michel parameter, $\varphi$, which improve the present limit on the deviation of this parameter from the standard model values. 


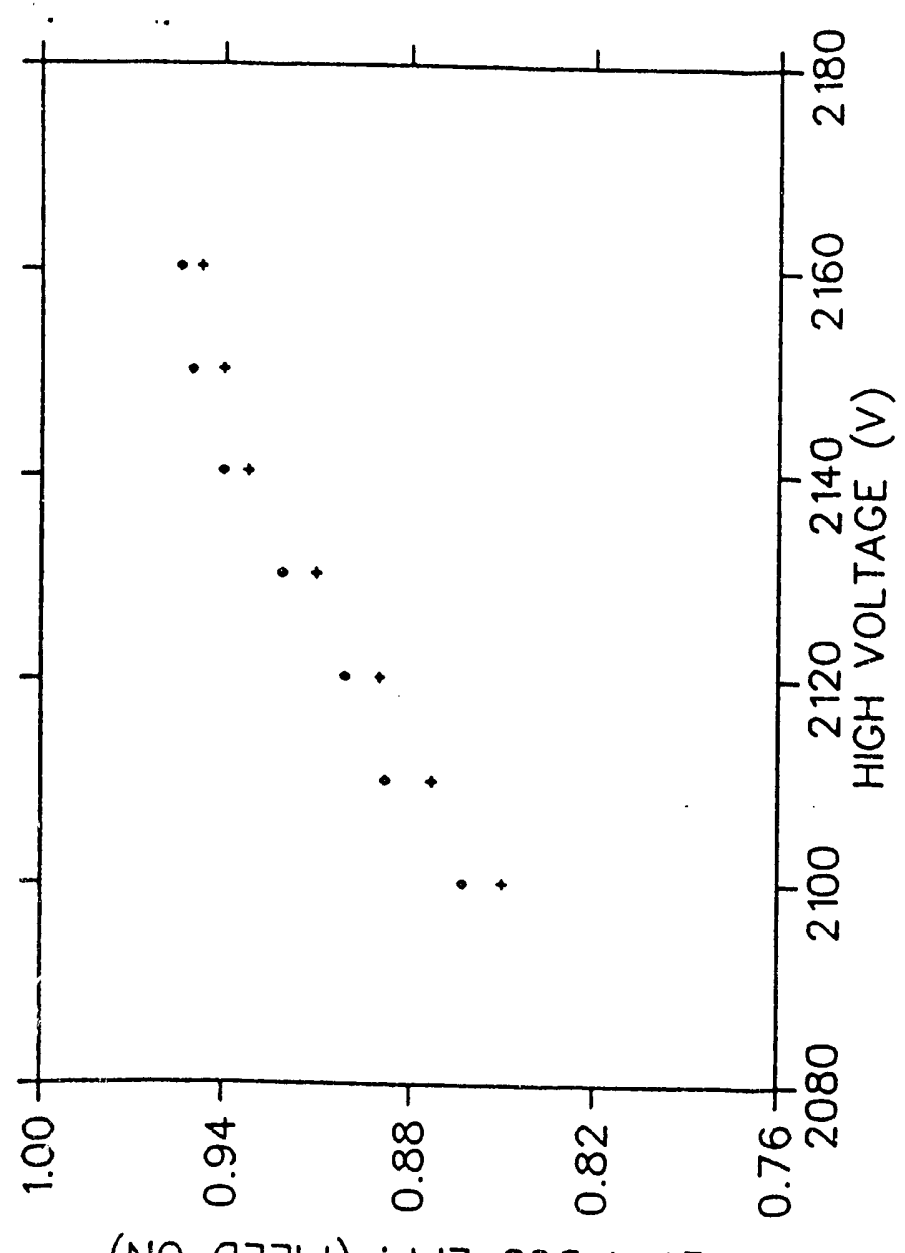

(NO ר א

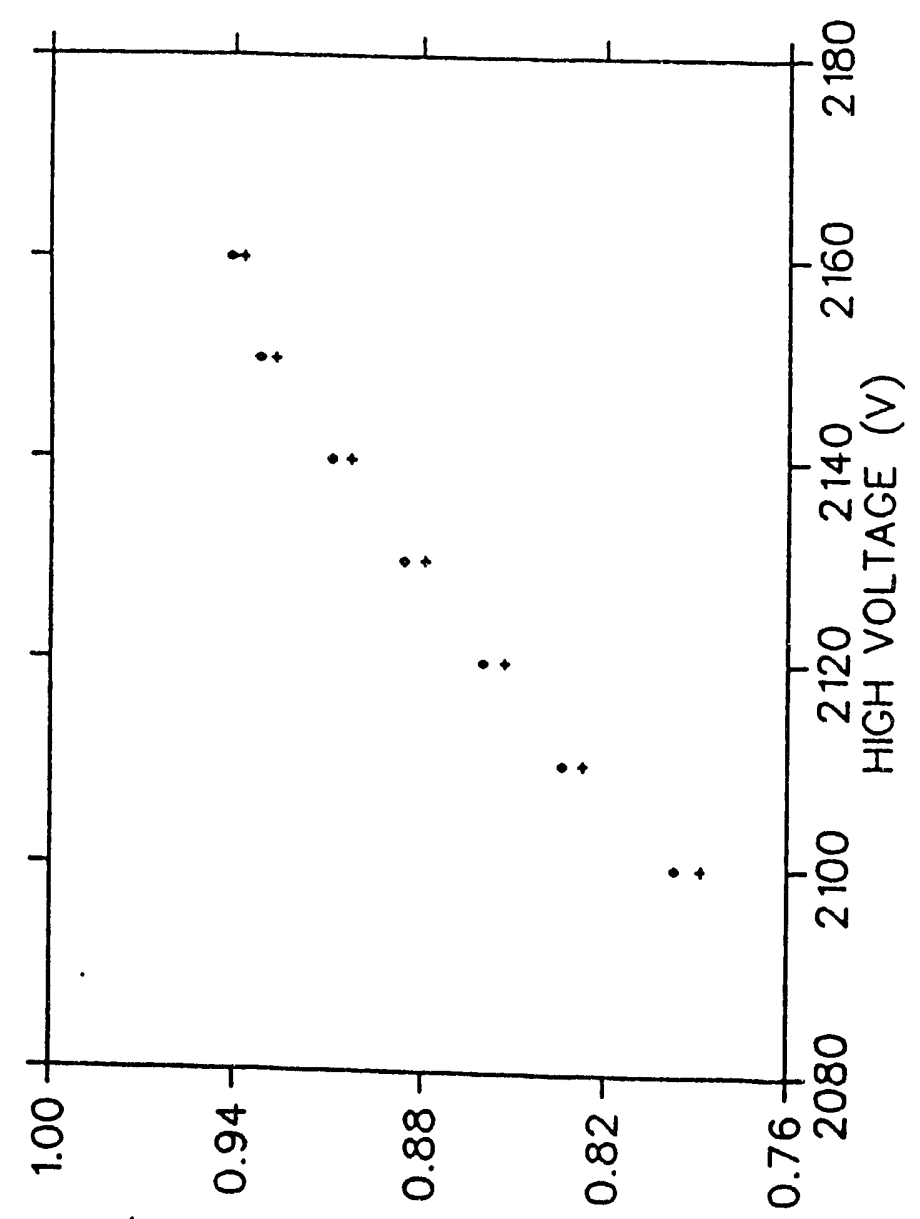

$\leftrightarrow \quad$ (NO 0า31 )

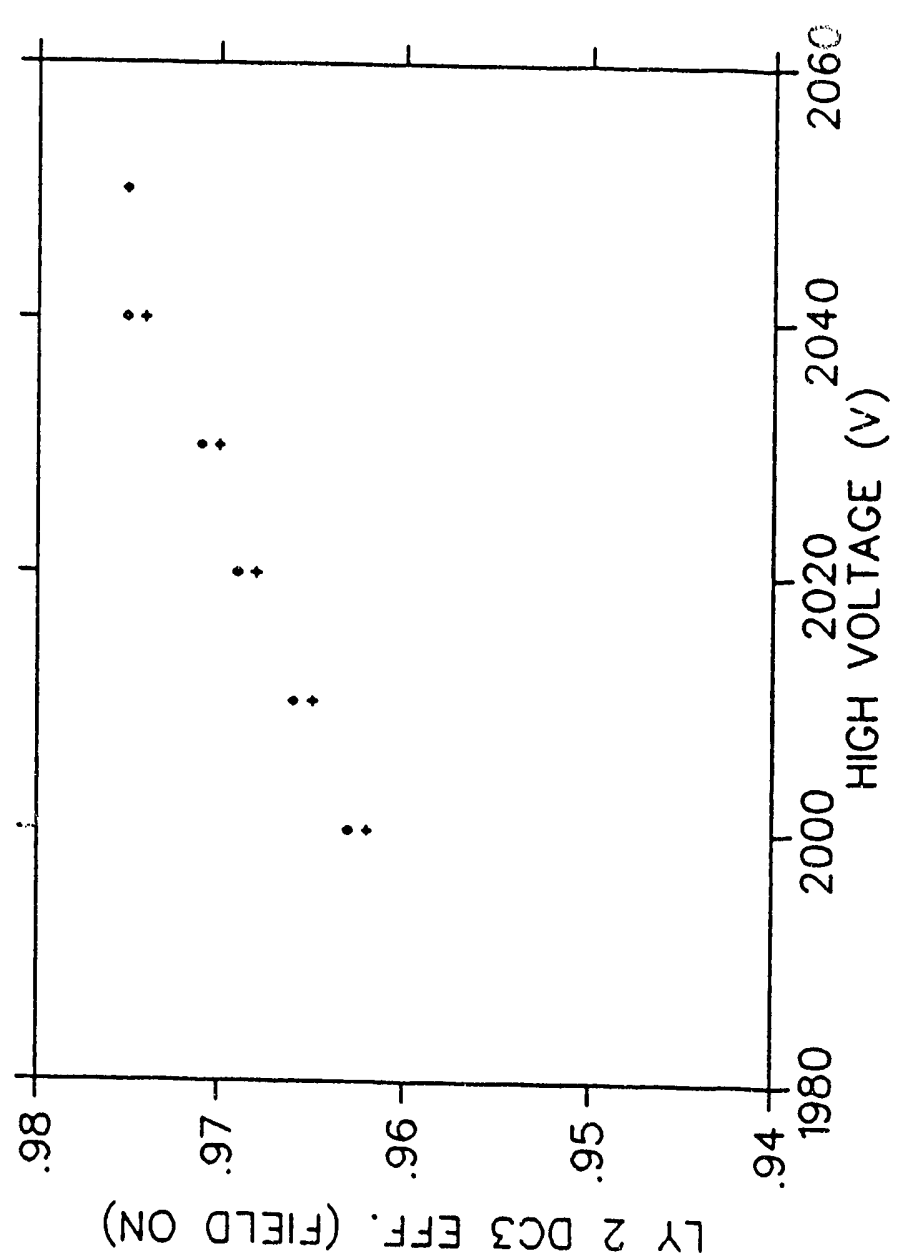

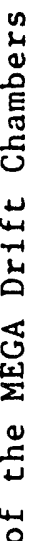

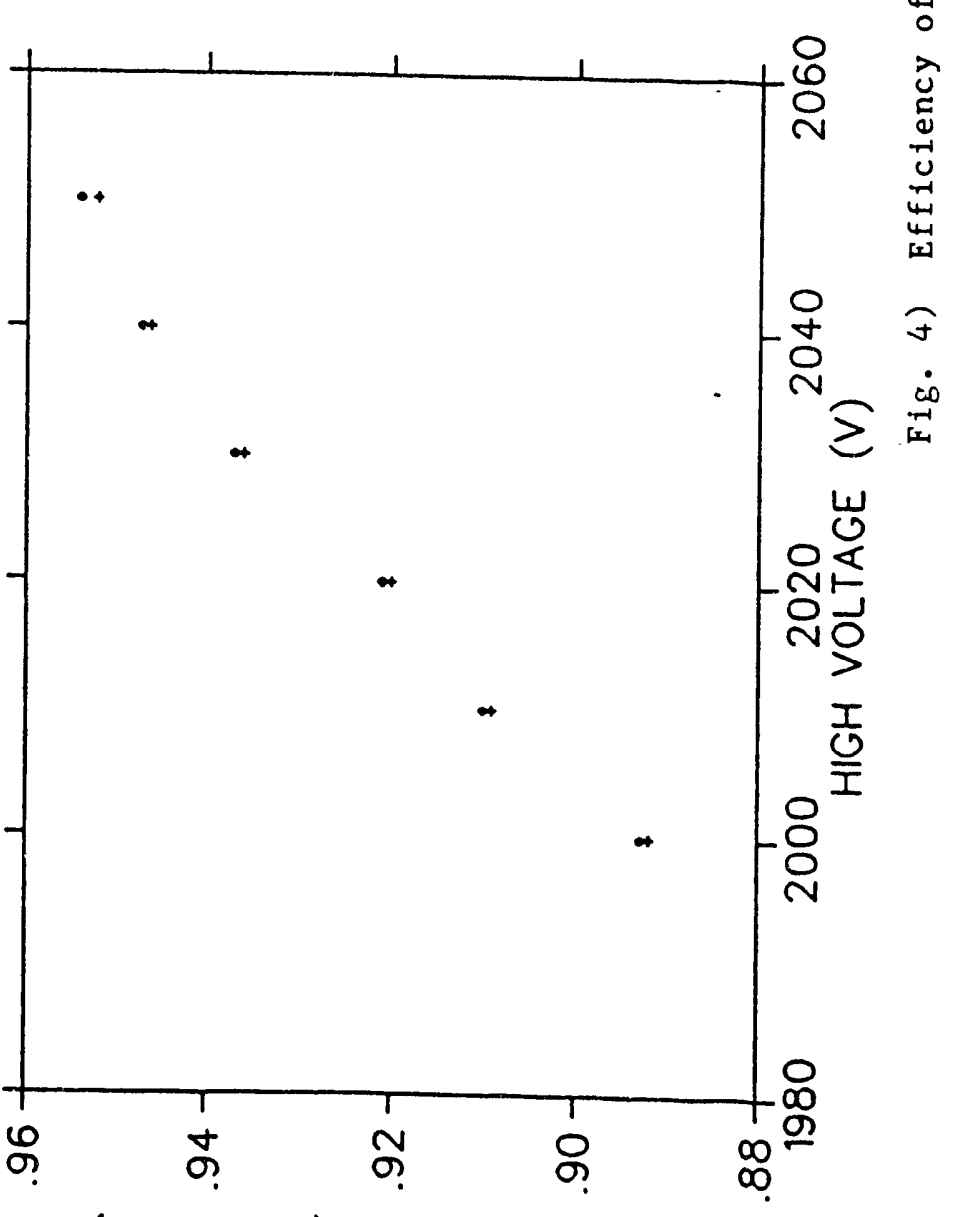

(NO 0า131) $9173200<17$ 


\section{Spin structure Function of Nucleons at CERN}

Figure 5 shows the layout of the spin Muon Collaboration (NA47) apparatus at CERN ${ }^{29}$. Over the past several years our group has played a principal role in the design and development of the ST67 streamer tube arrays used to track the muons behind the absorber wall. Our specific hardware contribution to the ST67 effort consists of the readout electronics including everything from the on-detector boards to the CAMAC modules.

These electronics were designed specifically for this application, and their delivery and installation took place this past spring prior to beam turn-on. The system includes 12,288 channels divided among 384 thirty-two channel boards. These are readout by 4 separate CAMAC modules through dedicated line driver power isolation modules. The design incorporates several new features over previous streamer tube readout systems. There is an onboard trigger coincidence for each group of 4 channels allowing one to significantly reduce background hits from out-of-time events.

The signals between the onboard electronics and the remote CAMAC crate are photo-optically decoupled so there are no common ground connections eliminating one persistent source of noise (due to ground loops). The shift register encoding is asynchronous with the shift pulse to allow arbitrary cable lengths and component locations. A fast trigger input was also provided to allow shorter onboard data storage time. 
SMC: FXPFRIMENT - SPF.'TROMEITER

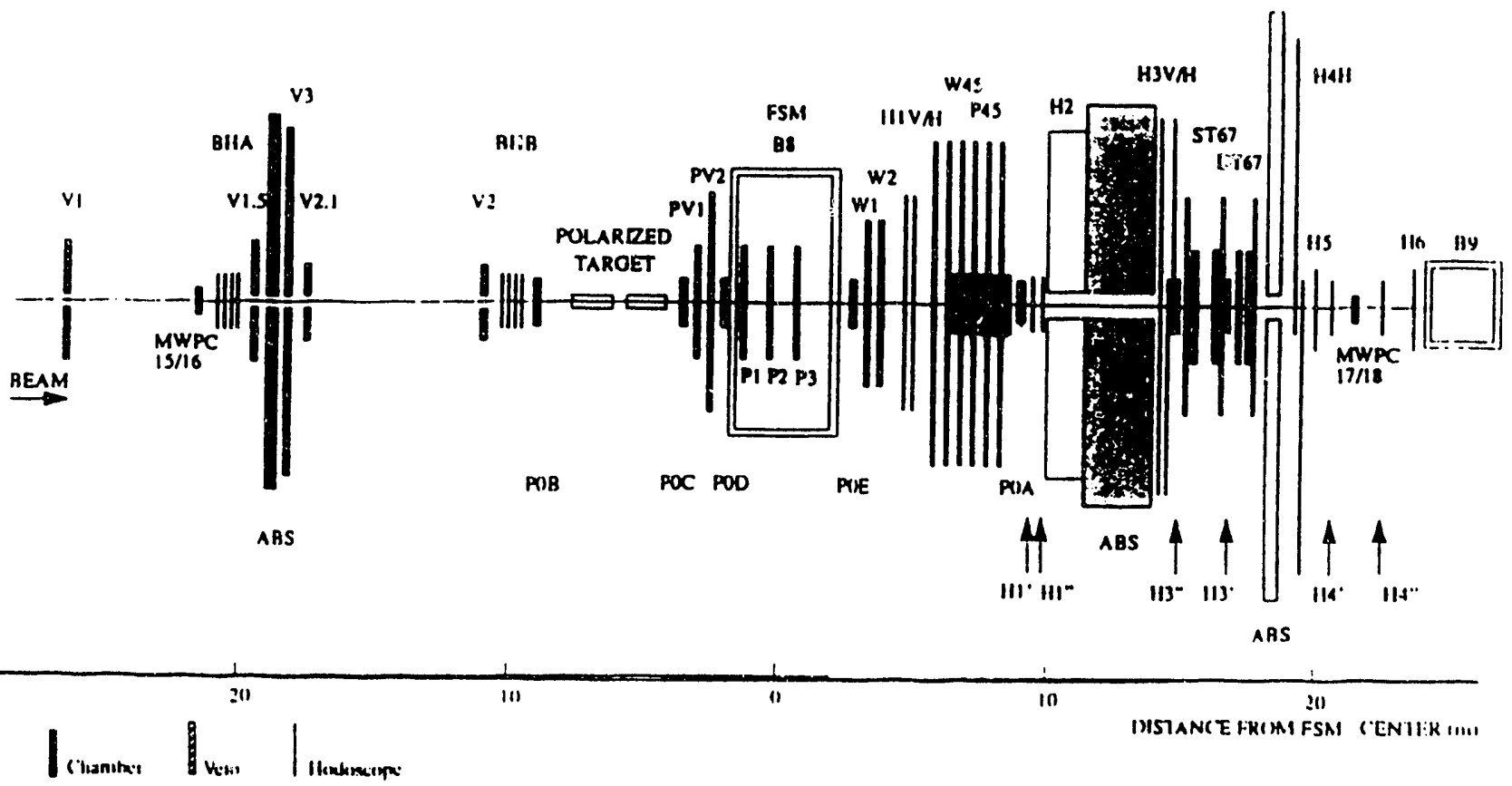

Fig. 5) Layout of the SMC Experiment 
These components were all received and installed over a 4 week period and have performed exceptionally well for 4 months. The entire ST67 detector system is now considered to be fully operational and in routine service. Sufficient spares are available to maintain the system in its present configuration for several years. ST67 is now the most stable component in the SMC spectrometer.

Figure 6 gives an example of the ST67 muon detector reconstructed track participation efficiency of each of the 32 coordinates in ST67. The maximum geometric efficiency for the streamer tubes is about $83 \%$ and the numbers are absolute efficiencies ( $i . e$. based or the number of valid triggers where all other indications predict that the triggering muon track should have been reconstructible by ST67.

Figure 7 shows a portion of the data to be obtained this year. Proton data will be taken during part of 1993, but significant results based on subtractions calculated from prior EMC proton data will yield the worlds first spin dependent neutron structure functions. Present plans are to run this apparatus for a minimum of 2 and very likely 3 years (through 1995).

\section{E. Instrumentation}

Several distinct problems with materials used in present wire chambers led us to initiate contact with the Textron specialty Materials Division in Lowell, Mass. In particular, three separate parameters were of interest. First, and most obvious is strength. This includes long term stability as well as strength to weight 


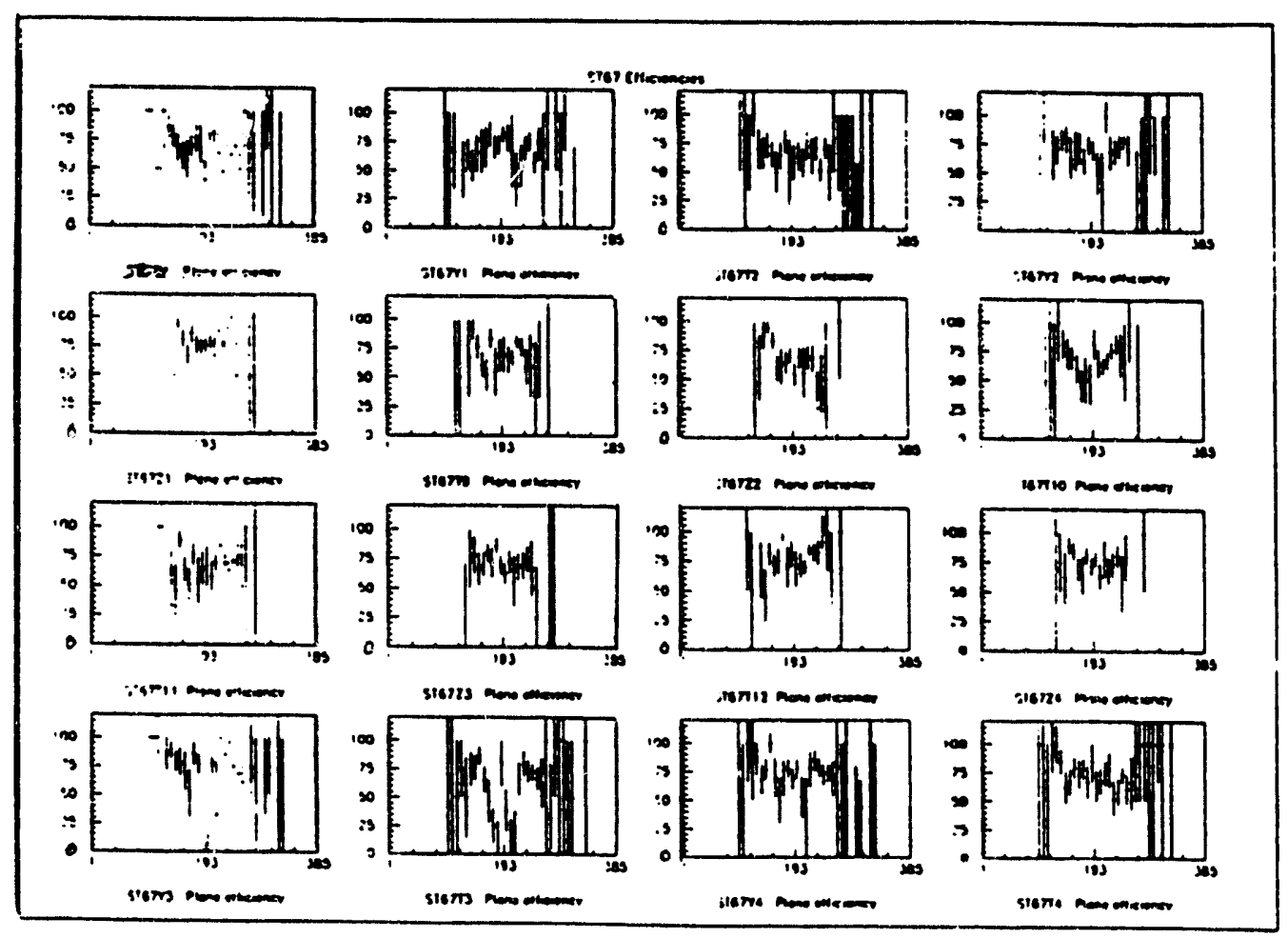

Fig. 6) Efficiency in the Muon trade reconstruction for the ST67 c) sunters 
Projected statistical errors for SMC data on deuteron in 1991,1992. and for EMC proton data.

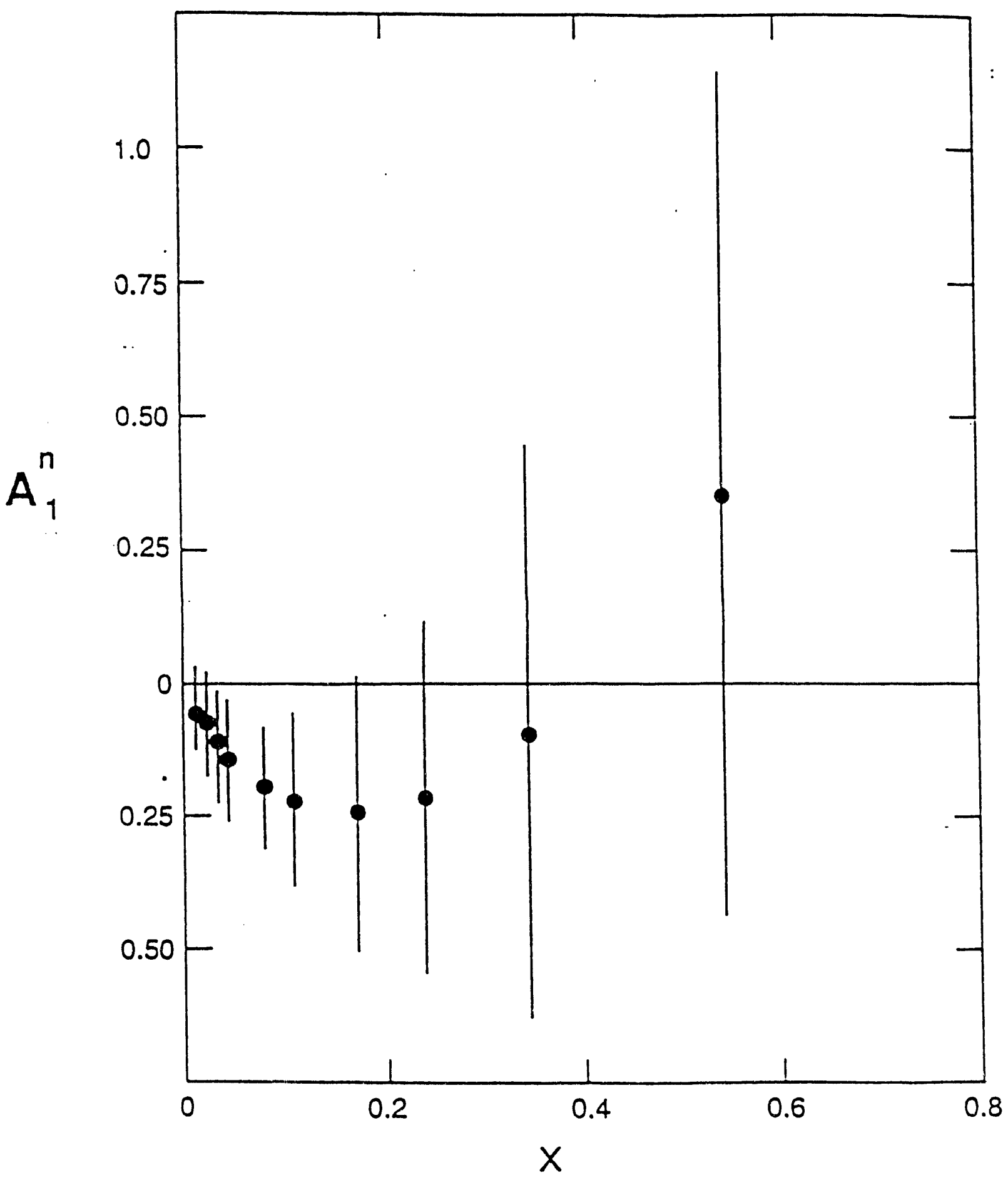

Fig. 7) Projected SMC data for the 1992 run 
ratio. Second is the mean atomic number. High atomic number elements like tungsten are a problem when chambers are used with low energy electrons. Boron wire in this case is significantly better than aluminum, and can be many times stronger. Finally, there is a desire to control the uniformity of the resistivity of the wire for charge division applications. Textron has, in the past, developed products that have great potential for use in wire chambers.

A meeting was organized at Textron's facility. This resulted in a Request for Proposal from us to which they subsequently responsed. In essence the present state of our program is that samples of wire of different types are being procured. (This is Phase I in the RFP and was funded by the SSC GEM muon group because of their interest in at least one of the samples). These samples represent the current off-of-the-shelf Textron capability and are in no way optimized for our intended use.

As described in the appendices, the three types of materials we wish to evaluate include: Silicon Carbide (SiC), which holds the promise of extreme strength to weight improvements over tungsten (by an order of magnitude) and a considerable improvement in surface quality which may improve total charge lifetime performance; Boron wire for use as field wire in drift chambers to reduce the low energy electron scattering; and finally precision Carbon Monofilament for use in charge division. All of these materials show considerable promise for vast improvement with a dedicated R\&D effort. 


\section{REFERENCES}

1) M. May, et al, Phys. Rev. Lett. 47 (1981) 1106; 51 (1983) 2085

R.E. Chrien, et a.l, Phys. Lett. 89 B (1979) 31

C. Milner, et al, Phys. Rev. Lett. 54 (1985) 1237

R. Grace, etal, Phys. Rev. Lett. $\underline{55}$ (1985) 1055

2) L. Biedenharn and E.V. Hungerford, Phys. Lett. 142B (1984) 232

3) A.R. Bodmer, Phys. Rev. 141 (1966) 1386

4) R.H. Dalitz, Nucl. Phys., Gordon and Breech, NY 1969

5) M. Bejidian, etal, Phys. Lett. $\underline{94 B}$ (1980) 480

6) P.J.G. Mulder, et al, Phys. Rev. D25 (1982) 1269; D26 (1982) 3039

7) A.T.M. Aerts, et al, Nucl. Phys. B253 (1985) 116

8) T.A. Griffy and R.J. Oakes, Phys. Rev. 135B (1964) 1161

9) C.B. Dover, Nucleonika 25 (1980) 521

10) E. Morris, dissertation Oxford Univ. 1974; S.T. Brown, et al, Nucl. Phys. B47 (1972) 138; R. Koaplus and L. Rodberg, Phys. Rev. 115 (1959) 1058

11) R. Bertini, et al, Phys. Lett. 90B (1980) 395

H. Piekarz, etal, Phys. Lett. $\frac{110 \mathrm{~B}}{\text { (1982) } 428}$

T. Yamazaki, etal, Phys. Rev. Lett. 54 (1985) 102

L.G. Tang, et al, Phys. Rev. C35 (1988) 846

R.E. Chrien, etal, Phys. Rev. C35 (1987) 1589

12) A. Gal and C. Dover, Phys. Rev. Lett 44 (1980) 379

13) R.S. Hayano, Phys. Lett. B231 (1989) 355

T. Harada and Y. Akaishi, Phys. Lett. 262B (1991) 205

14) C.B. Dover and G.E. Walker, Phys. Rev. C19, 1393 (1979)

S.R. Cotanch, Phys. Rev. C18, 1941 (1978); Nucl. Phys.

A308, 253 (1978); Phys. Rev. C21, 2115 (1980)

M.J. Paez and R.H. Landau, Phys. Rev. C24, 1120 (1981)

15) P.B. Siegel, et al, Phys. Rev. C30, 1256 (1984)

16) R. Sawafta, et al, Phys. Rev. Lett. to be published

17) D. Marlow, et al, Phys. Rev. C25, 2619 
18) P.B. Siegel, W.B. Kaufman and W.R. Gibbs, Phys. Rev. C31, 2184

19) G.E. Brown, etal, Phys. Rev. Lett. 60,2723

20) G.E. Brown, et al, preprint

21) M.M. Nagels, et al, Arrn. Phys. (NY) 79 (1973) 388, Phys. Rev. D15 (1977) 2547, Phys. Rev. D20 (1979) 1633,

P.M. Maessen, et al, Phys. Rev. C40 (1989) 2226

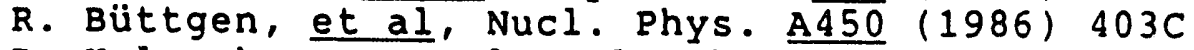

B. Holzenkamp, et al Nucl. Phys. A500 (1989) 485

K. Holinde, Nucl. Phys. A479 (1988) 197c

22) D.H. Davis, AIP Conf. Proc. $\underline{224}$ (1991) 38

23) K. Maltman, AIP Conf. Proc. 224 (1991) 211

24) H. Bando, T. Motoba, and J. Zofka J. Mod. Phys. A5 (1990) 1

25) C.B. Dover and D.J. Millener, BNL 44651, May 1990

T.W. Donnelly and S.R. Cotanch, Proceedings of the 1985 CEBAF Summer Study, Jan. 1986

26) R.D. Bolton, et al, Phys. Rev. Lett. 19 (1984) 1415

27) R.D. Bolton, et al, Nucl. Inst. and Meth. A241 (1985) 52

G. Aiken, et al, SLAC-PUB- 2642

H. Van der Graaf and J.P. Wagenaar, Nucl. Inst. and Meth. 217 (1983) 330

V. Radeka and P. Rehak, IEEE Nucl. Sci., $\underline{25}$ (1978) 1

28) A.R. Erwin, et al, Nucl. Inst. and Meth. A237 (1985) 493

29) See SMC proposal submitted to DOE 
1) "Defect Size Dependence of Critical Current Density Enhancement for Irradiated $\mathrm{YBa}_{2} \mathrm{Cu}_{3} \mathrm{O}_{7} "$, J. Bechtold, et al, Physica $\mathrm{C197}$ (1992) 199

2) "Irradiation Effects on Flux Pinning and $\mathrm{J}^{c}$ in High Temperature superconductors", J. Bechtold, etal, Physica C185 (1991) 2211

3) "Measurement of the Influence of Nuclear Density on $\mathrm{K}^{+}$Total Cross Sections", R. Sawafta, et al Phys. Rev. Lett.

4) "Pion Production at Small Angles", J. Langenbrunner, et al, Nucl. Inst. and Meth., to be published

5) Search for A strangeness - 1 Dibaryon below the $\Sigma N$ Threshold, K. Johnston, et al, Phys. Rev. C, to be published

\section{DISSERTATIONS}

1) Mohamad Barakat, "Search for $\Sigma$ Hypernuclear states in the $A=3,4$ systems", July 1992

2) Myonggwon Park, "Ions from a LASER Accelerated Plasma", September 1992

3) Ioannis Tzamouranis, "Nuclear Effects Observed in a Measurement of the Nuclear structure Function Ratio of $T$ in and Carbon and a Comparison to other Experiments and current Theoretical Models", May 1992 
1) "Strange Things are Happening", E. Hungerford, Texas A\&M University

2) "Hypernuclear Physics", E. Hungerford, Trinity University

3) "Strange Things are Happening", E. Hungerford, University of Houston

4) "Recent Discoveries in Cosmic Background Radiation", L. Pinsky, University of Houston-Downtown 

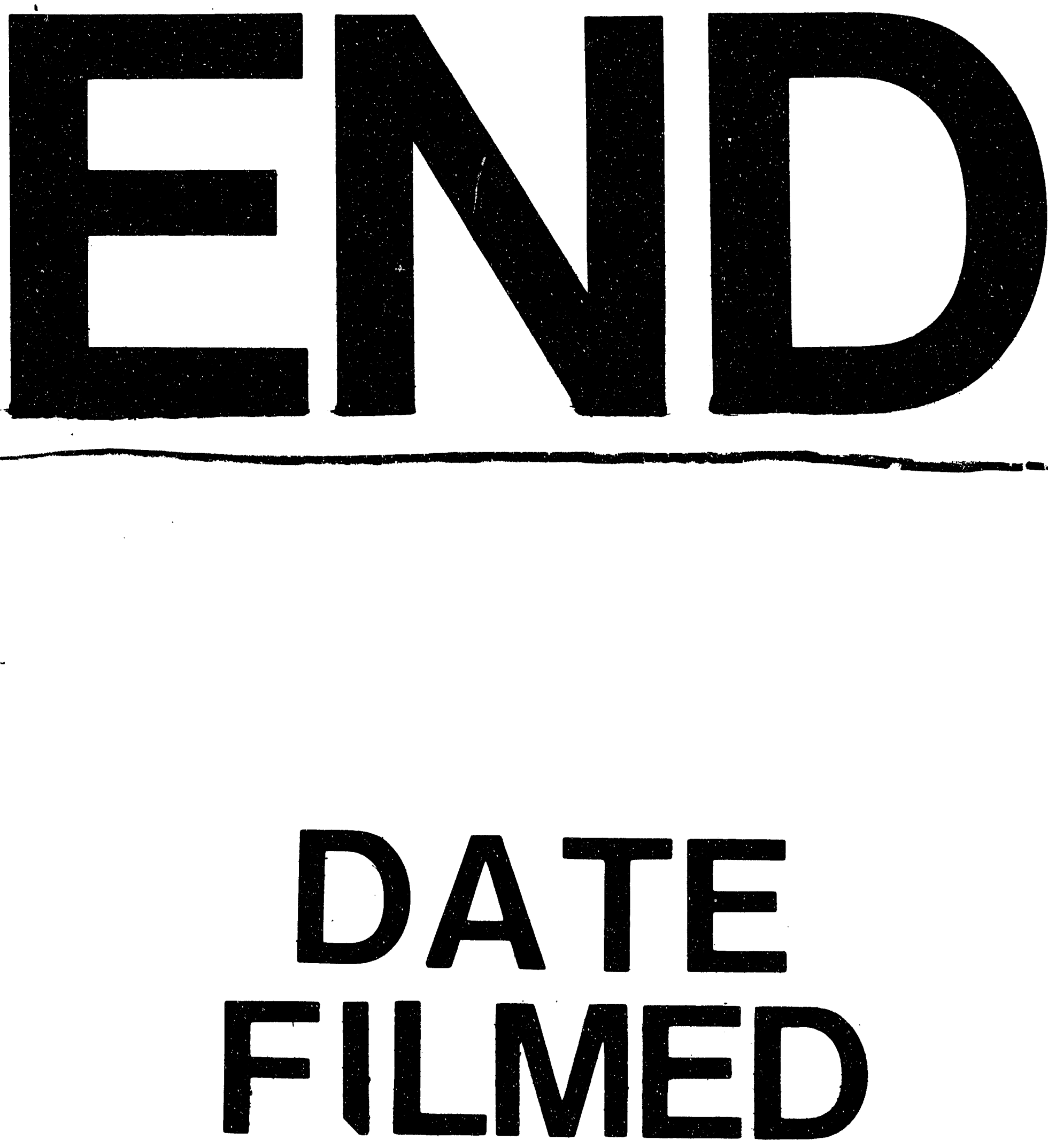

\begin{tabular}{l|l|l}
4 & $28 / 93$
\end{tabular} 
